TRANSACTIONS OF THE

AMERICAN MATHEMATICAL SOCIETY

Volume 364, Number 1, January 2012, Pages 119-136

S 0002-9947(2011)05269-4

Article electronically published on August 9, 2011

\title{
SOME EIGENVALUE PROBLEMS FOR VECTORIAL STURM-LIOUVILLE EQUATIONS WITH EIGENPARAMETER DEPENDENT BOUNDARY CONDITIONS
}

\author{
CHI-HUA CHAN
}

\begin{abstract}
We investigate the $n$-dimensional vectorial Sturm-Liouville equation

$$
\vec{y}^{\prime \prime}(x)+\left[\lambda^{2} I_{n}-Q(x)\right] \vec{y}(x)=\overrightarrow{0}
$$

with eigenparameter dependent boundary conditions

$$
\vec{y}(0)=\overrightarrow{0}, A \vec{y}^{\prime}(\pi)+\lambda \vec{y}(\pi)=\overrightarrow{0} .
$$

Under the assumption that $Q(x)$ is nonnegative definite in $[0, \pi]$, we prove that the eigenvalues of the $n$-dimensional vectorial Sturm-Liouville equation are real.

For the case $n=2$, we show that the algebraic multiplicity of an eigenvalue of the problem as a zero of the characteristic function

$$
\omega_{A}(\lambda ; Q)=\operatorname{det}\left[A Y^{\prime}\left(\pi ; \lambda^{2} ; Q\right)+\lambda Y\left(\pi ; \lambda^{2} ; Q\right)\right]
$$

is equal to its geometric multiplicity. By the theory of Hadamard's factorization, we also prove that the characteristic function $\omega_{A}(\lambda ; Q)$ is uniquely determined by the spectral set of the equation. Moreover, we consider the inverse problem for the equation, i.e., how many spectral sets can determine the potential function $Q(x)$ uniquely, and we find that three spectral sets are necessary for us to determine the potential function $Q(x)$ uniquely.
\end{abstract}

\section{INTRODUCTION}

The purpose of this paper is to investigate a class of vectorial Sturm-Liouville equations with eigenparameter dependent boundary conditions. The spectral problems related to the Sturm-Liouville equations with eigenparameter dependent boundary conditions were first systematically studied by Hochstadt in $[\mathbf{H}]$. There, Hochstadt investigated this kind of eigenvalue problem for Sturm-Liouville equations with a scalar coefficient. He considered the following eigenvalue problem:

$$
\left\{\begin{array}{l}
y^{\prime \prime}(x)+\left[\lambda^{2}-q(x)\right] y(x)=0,0 \leq x \leq \pi, \\
y(0)=0, a y^{\prime}(\pi)+\lambda y(\pi)=0
\end{array}\right.
$$

where $a \neq 0, a \in \mathbb{R}, q(x)$ is real valued and integrable on $[0, \pi]$. Let $y(x ; \nu ; q)$ denote the solution of the initial-value problem

$$
y^{\prime \prime}(x)+[\nu-q(x)] y(x)=0, y(0)=0, y^{\prime}(0)=1 \text {. }
$$

Received by the editors August 27, 2009 and, in revised form, December 8, 2009.

2010 Mathematics Subject Classification. Primary 34B08.

(C)2011 American Mathematical Society Reverts to public domain 28 years from publication 
Denote

$$
\omega_{a}(\lambda ; q)=a y^{\prime}\left(\pi ; \lambda^{2} ; q\right)+\lambda y\left(\pi ; \lambda^{2} ; q\right)
$$

and call it the characteristic function of equation (1.1). Then $\lambda_{*}$ is an eigenvalue of equation (1.1) if and only if $\lambda_{*}$ is a zero of $\omega_{a}(\lambda ; q)$. The number of linearly independent solutions corresponding to $\lambda_{*}$ is called the geometric multiplicity of $\lambda_{*}$, and the multiplicity of $\lambda_{*}$ as a zero of $\omega_{a}(\lambda ; q)$ is called the algebraic multiplicity of $\lambda_{*}$.

Under some assumptions, Hochstadt used the idea of inverse Liouville transformation to transform equation (1.1) into a string equation of the form

$$
\left\{\begin{array}{l}
z^{\prime \prime}(x)+\lambda^{2} M^{4}(x) z(x)=0,0 \leq x \leq c, \\
z(0)=0, a z^{\prime}(c)+\lambda z(c)=0
\end{array}\right.
$$

where $c=\int_{0}^{\pi} \frac{1}{M^{2}(t)} d t$. Then he transformed equation (1.2) into an integral equation and applied the properties of the integral operator corresponding to the integral equation to obtain the following result:

Theorem 1.1. All eigenvalues of equation (1.1) are real, geometrically simple, and algebraically simple.

Following Theorem 1.1, we see that the characteristic function $\omega_{a}(\lambda ; q)$ of equation (1.1) is completely determined by its eigenvalues. Moreover, Hochstadt proved in $[\mathrm{H}]$ that the potential function of equation (1.1) is uniquely determined by the spectrum of equation $(1.1)([\mathrm{H}$, Theorem B] $)$ :

Theorem 1.2. Consider the boundary value problems

$$
\begin{aligned}
& y^{\prime \prime}(x)+\left[\lambda^{2}-q_{1}(x)\right] y(x)=0, y(0)=a y^{\prime}(\pi)+\lambda y(\pi)=0, \\
& u^{\prime \prime}(x)+\left[\lambda^{2}-q_{2}(x)\right] u(x)=0, u(0)=a u^{\prime}(\pi)+\lambda u(\pi)=0,
\end{aligned}
$$

where $a \neq 0, a \in \mathbb{R}, q_{j}(x)$ are real valued and integrable on $[0, \pi], j=1,2$. If $\omega_{a}\left(\lambda ; q_{1}\right)=\omega_{a}\left(\lambda ; q_{2}\right)$, then $q_{1}=q_{2}$ almost everywhere in $[0, \pi]$.

We are interested in extending Hochstadt's work to the $n$-dimensional vectorial Sturm-Liouville equations of the form

$$
\left\{\begin{array}{l}
\vec{y}^{\prime \prime}(x)+\left[\lambda^{2} I_{n}-Q(x)\right] \vec{y}(x)=\overrightarrow{0}, \\
\vec{y}(0)=\overrightarrow{0}, A \vec{y}^{\prime}(\pi)+\lambda \vec{y}(\pi)=\overrightarrow{0},
\end{array}\right.
$$

where $Q(x)$ is an $n \times n$ real symmetric matrix-valued smooth function defined in the interval $[0, \pi], A$ is an $n \times n$ nonsingular real symmetric matrix, $\vec{y}(x)$ is an $\mathbb{R}^{n}$ function defined in the interval $[0, \pi]$, and $\overrightarrow{0}$ is the zero vector in $\mathbb{R}^{n}$. Let $Y(x ; \nu ; Q)$ denote the $n \times n$ matrix solution of the $n \times n$ matrix differential equation

$$
Y^{\prime \prime}(x)+\left[\nu I_{n}-Q(x)\right] Y(x)=0, Y(0)=0, Y^{\prime}(0)=I_{n},
$$

where 0 is the $n \times n$ zero matrix. Denote

$$
\omega_{A}(\lambda ; Q)=\operatorname{det}\left(A Y^{\prime}\left(\pi ; \lambda^{2} ; Q\right)+\lambda Y\left(\pi ; \lambda^{2} ; Q\right)\right),
$$

and call it the characteristic function of equation (1.3). Then $\lambda_{*}$ is an eigenvalue of equation (1.3) if and only if $\lambda_{*}$ is a zero of $\omega_{A}(\lambda ; Q)$. The number of linearly independent solutions corresponding to $\lambda_{*}$ is called the geometric multiplicity of $\lambda_{*}$, and the multiplicity of $\lambda_{*}$ as a zero of $\omega_{A}(\lambda ; Q)$ is called the algebraic multiplicity of $\lambda_{*}$. 
However, there are some major differences between the scalar case considered by Hochstadt and its vectorial analogy. We find a class of matrix potential functions for which the equation possesses nonreal eigenvalues. Consider the following example: assume $\frac{1}{4}<a<\frac{9}{4}, b \geq 0$ and consider the eigenvalue problem

$$
\left\{\begin{array}{l}
\vec{y}^{\prime \prime}(x)+\left[\lambda^{2} I_{2}-\left(\begin{array}{cc}
b & 0 \\
0 & -a
\end{array}\right)\right] \vec{y}(x)=\overrightarrow{0} \\
\vec{y}(0)=\overrightarrow{0},\left(\begin{array}{ll}
0 & 1 \\
1 & 0
\end{array}\right) \vec{y}^{\prime}(\pi)+\lambda \vec{y}(\pi)=\overrightarrow{0}
\end{array}\right.
$$

where $\vec{y}(x)$ is an $\mathbb{R}^{2}$-function defined in the interval $[0, \pi]$, and $\overrightarrow{0}$ is the zero vector in $\mathbb{R}^{2}$. Denote

then we find

$$
Q_{1}=\left(\begin{array}{cc}
b & 0 \\
0 & -a
\end{array}\right), A_{1}=\left(\begin{array}{ll}
0 & 1 \\
1 & 0
\end{array}\right) \text {, }
$$

$$
Y\left(x ; \lambda^{2} ; Q_{1}\right)=\left(\begin{array}{cc}
\frac{\sin \left(\sqrt{\lambda^{2}-b} x\right)}{\sqrt{\lambda^{2}-b}} & 0 \\
0 & \frac{\sin \left(\sqrt{\lambda^{2}+a} x\right)}{\sqrt{\lambda^{2}+a}}
\end{array}\right) .
$$

Thus the characteristic function $\omega_{A_{1}}\left(\lambda ; Q_{1}\right)$ of equation (1.4) is

$$
\begin{aligned}
\omega_{A_{1}}\left(\lambda ; Q_{1}\right) & =\operatorname{det}\left(A_{1} Y^{\prime}\left(\pi ; \lambda^{2} ; Q_{1}\right)+\lambda Y\left(\pi ; \lambda^{2} ; Q_{1}\right)\right) \\
& =\frac{\lambda^{2}}{\sqrt{\lambda^{2}-b} \sqrt{\lambda^{2}+a}} \sin \left(\sqrt{\lambda^{2}-b} \pi\right) \sin \left(\sqrt{\lambda^{2}+a} \pi\right) \\
& -\cos \left(\sqrt{\lambda^{2}-b} \pi\right) \cos \left(\sqrt{\lambda^{2}+a} \pi\right) .
\end{aligned}
$$

Taking $\lambda=c i$ in equation (1.6), where $c>0, i=\sqrt{-1}$, we have

$$
\begin{aligned}
\omega_{A_{1}}\left(c i ; Q_{1}\right) & =-\frac{c^{2}}{\sqrt{b+c^{2}} \sqrt{a-c^{2}}} \sinh \left(\sqrt{b+c^{2}} \pi\right) \sin \left(\sqrt{a-c^{2}} \pi\right) \\
& -\cosh \left(\sqrt{b+c^{2}} \pi\right) \cos \left(\sqrt{a-c^{2}} \pi\right) .
\end{aligned}
$$

Note that

$$
\lim _{c \rightarrow 0^{+}} \omega_{A_{1}}\left(c i ; Q_{1}\right)=-\cosh (\sqrt{b} \pi) \cos (\sqrt{a} \pi)>0
$$

and

$$
\lim _{c \rightarrow \sqrt{a}^{-}} \omega_{A_{1}}\left(c i ; Q_{1}\right)=-\frac{a \pi}{\sqrt{b+a}} \sinh (\sqrt{b+a} \pi)-\cosh (\sqrt{b+a} \pi)<0 .
$$

From (1.8) and (1.9), we find that there exists $c_{0}$ in $(0, \sqrt{a})$ such that $\omega_{A_{1}}\left(c_{0} i ; Q_{1}\right)=$ 0 , i.e., $c_{0} i$ is an eigenvalue of equation (1.4). This example shows that there are nonpositive definite and indefinite potential functions $Q(x)$ such that equation (1.3) has a nonreal eigenvalue.

Besides, the method of the inverse Liouville transformation used in Hochstadt's work for proving Theorem 1.1 cannot be extended to the vectorial case. To remedy these situations, in section 3 , we use a different approach to deal with the vectorial case under the assumption that the potential function $Q(x)$ is nonnegative definite for all $x$ in $[0, \pi]$. Under this assumption, we prove that the eigenvalues of equation (1.3) are real (see Theorem 3.1). The problem about the equality between the geometric multiplicity and the algebraic multiplicity of the eigenvalues of the vectorial Sturm-Liouville equation is more complicated. To deal with such a problem, 
we apply an approach similar to that used in $[\mathbf{S h}$. In $[\underline{S h}$, Shen considered the eigenvalue problems

$$
\begin{aligned}
& \left\{\begin{array}{l}
\vec{y}^{\prime \prime}(x)+\left[\lambda I_{2}-Q(x)\right] \vec{y}(x)=\overrightarrow{0}, 0 \leq x \leq 1, \\
\vec{y}(0)=\overrightarrow{0}, \vec{y}(1)=\overrightarrow{0},
\end{array}\right. \\
& \left\{\begin{array}{l}
\vec{z}^{\prime \prime}(x)+\left[\lambda I_{2}-Q(x)\right] \vec{z}(x)=\overrightarrow{0}, 0 \leq x \leq 1, \\
\vec{z}^{\prime}(0)=\overrightarrow{0}, \vec{z}(1)=\overrightarrow{0},
\end{array}\right.
\end{aligned}
$$

and

$$
\left\{\begin{array}{l}
\vec{u}^{\prime \prime}(x)+\left[\lambda I_{2}-Q(x)\right] \vec{u}(x)=\overrightarrow{0}, 0 \leq x \leq 1, \\
\vec{u}^{\prime}(0)-B \vec{u}(0)=\overrightarrow{0}, \vec{u}(1)=\overrightarrow{0},
\end{array}\right.
$$

where $Q(x)$ is a two-by-two real symmetric matrix-valued smooth function defined in $[0,1] ; B$ is a two-by-two nonsingular real symmetric matrix; $\vec{y}(x), \vec{z}(x), \vec{u}(x)$ are $\mathbb{R}^{2}$-functions defined in the interval $[0,1]$; and $\overrightarrow{0}$ is the zero vector in $\mathbb{R}^{2}$. He proved that if $\lambda_{*}$ is an eigenvalue of equation (1.10) (or (1.11), (1.12)), then the geometric multiplicity and the algebraic multiplicity of $\lambda_{*}$ are the same. On the other hand, let $\sigma_{D}(Q), \sigma_{N D}(Q)$, and $\sigma_{B}(Q)$ denote the spectral set of equations (1.10), (1.11), and (1.12), respectively. Shen also proved that if $B_{1}, B_{2}$, and $B_{3}$ are three linearly independent real symmetric matrices, then $\sigma_{D}(Q), \sigma_{N D}(Q)$, and $\sigma_{B_{j}}(Q), j=1,2$, 3 , determine $Q(x)$ uniquely.

In section 4 , we concentrate on the two-dimensional case in order to obtain some results about the geometric multiplicity and the algebraic multiplicity of an eigenvalue. Consider the eigenvalue problem

$$
\left\{\begin{array}{l}
\vec{y}^{\prime \prime}(x)+\left[\lambda^{2} I_{2}-Q(x)\right] \vec{y}(x)=\overrightarrow{0}, 0 \leq x \leq \pi, \\
\vec{y}(0)=\overrightarrow{0}, A \vec{y}^{\prime}(\pi)+\lambda \vec{y}(\pi)=\overrightarrow{0},
\end{array}\right.
$$

where $Q(x)$ is a two-by-two nonnegative definite matrix-valued smooth function defined in $[0, \pi], A$ is a two-by-two nonsingular real symmetric matrix, $\vec{y}(x)$ is an $\mathbb{R}^{2}$-function defined in the interval $[0, \pi]$, and $\overrightarrow{0}$ is the zero vector in $\mathbb{R}^{2}$. According to the results that we obtained in section 3 and using the argument similar to that Shen used to deal with equations (1.10), (1.11), and (1.12), we find that the geometric multiplicity and the algebraic multiplicity of an eigenvalue of equation (1.13) are the same (see Theorem 4.5). Applying this result and using some asymptotic arguments, we also prove that $\omega_{A}(\lambda ; Q)$ is uniquely determined by the spectral set of equation (1.13).

At the end of this paper, we study an inverse problem for the vectorial SturmLiouville equation with eigenparameter dependent boundary conditions: how many spectral sets of the eigenvalue problem of the form like equation (1.13) can determine $Q(x)$ uniquely? We find that the spectral sets corresponding to three linearly independent real symmetric matrices can determine $Q(x)$ uniquely (see Theorem 4.9). From the example we constructed in section 4 (see equations (4.41) and (4.42)), we find the requirement of three spectral conditions in Theorem 4.9 is optimal for the two-dimensional vectorial Hochstadt problem.

\section{Preliminaries}

In this section, we recall some properties which will be used later. Let $Q(x)$ be an $n \times n$ real symmetric matrix-valued smooth function defined in $[0, \pi]$, and 
let $A$ be an $n \times n$ nonsingular real symmetric matrix, where $n \in \mathbb{N}$. Consider the eigenvalue problem

$$
\left\{\begin{array}{l}
\vec{y}^{\prime \prime}(x)+\left[\lambda^{2} I_{n}-Q(x)\right] \vec{y}(x)=\overrightarrow{0}, \\
\vec{y}(0)=\overrightarrow{0}, A \vec{y}^{\prime}(\pi)+\lambda \vec{y}(\pi)=\overrightarrow{0},
\end{array}\right.
$$

where $\vec{y}(x)$ is an $\mathbb{R}^{n}$-function defined in the interval $[0, \pi]$, and $\overrightarrow{0}$ is the zero vector in $\mathbb{R}^{n}$. In order to investigate equation (2.1), we apply the following $n \times n$ matrix differential equations:

$$
\begin{aligned}
Y^{\prime \prime}(x)+\left[\nu I_{n}-Q(x)\right] Y(x) & =0, Y(0)=0, Y^{\prime}(0)=I_{n}, \\
Z^{\prime \prime}(x)+\left[\nu I_{n}-Q(x)\right] Z(x) & =0, Z(0)=I_{n}, Z^{\prime}(0)=0,
\end{aligned}
$$

where 0 is the $n \times n$ zero matrix. Let $Y(x ; \nu ; Q)$ and $Z(x ; \nu ; Q)$ denote the $n \times n$ matrix solutions of equations (2.2) and (2.3), respectively. Then we have (see [PT], $[\mathrm{Sh}]$ )

Lemma 2.1. For fixed $x \in[0, \pi]$, the matrix functions $Y\left(x ; \lambda^{2} ; Q\right), Y^{\prime}\left(x ; \lambda^{2} ; Q\right)$, $Z\left(x ; \lambda^{2} ; Q\right)$, and $Z^{\prime}\left(x ; \lambda^{2} ; Q\right)$ are entire functions with respect to variable $\lambda^{2}$, and

$$
\begin{aligned}
& Y\left(x ; \lambda^{2} ; Q\right)=\frac{\sin \sqrt{\lambda^{2}} x}{\sqrt{\lambda^{2}}} I_{n}+O\left(\frac{e^{\left|\operatorname{Im} \sqrt{\lambda^{2}}\right| x}}{|\lambda|^{2}}\right),|\lambda| \rightarrow \infty, \\
& Y^{\prime}\left(x ; \lambda^{2} ; Q\right)=\left(\cos \sqrt{\lambda^{2}} x\right) I_{n}+O\left(\frac{e^{\left|\operatorname{Im} \sqrt{\lambda^{2}}\right| x}}{|\lambda|}\right),|\lambda| \rightarrow \infty, \\
& Z\left(x ; \lambda^{2} ; Q\right)=\left(\cos \sqrt{\lambda^{2}} x\right) I_{n}+O\left(\frac{e^{\left|\operatorname{Im} \sqrt{\lambda^{2}}\right| x}}{|\lambda|}\right),|\lambda| \rightarrow \infty, \\
& Z^{\prime}\left(x ; \lambda^{2} ; Q\right)=-\left(\sqrt{\lambda^{2}} \sin \sqrt{\lambda^{2}} x\right) I_{n}+O\left(e^{\left|\operatorname{Im} \sqrt{\lambda^{2}}\right| x}\right),|\lambda| \rightarrow \infty .
\end{aligned}
$$

It is easy to see that $\lambda_{*}$ is an eigenvalue of equation (2.1) if and only if

$$
\operatorname{det}\left(A Y^{\prime}\left(\pi ; \lambda_{*}^{2} ; Q\right)+\lambda_{*} Y\left(\pi ; \lambda_{*}^{2} ; Q\right)\right)=0 .
$$

Denote

$$
\omega_{A}(\lambda ; Q)=\operatorname{det}\left(A Y^{\prime}\left(\pi ; \lambda^{2} ; Q\right)+\lambda Y\left(\pi ; \lambda^{2} ; Q\right)\right) .
$$

Then $\omega_{A}(\lambda ; Q)$ is an entire function of order one with respect to $\lambda$, which shall be called the characteristic function of equation (2.1). Furthermore, by (2.4) and $(2.5), \omega_{A}(\lambda ; Q)$ has the following asymptotic formula for the case $n=2$ :

$$
\begin{aligned}
\omega_{A}(\lambda ; Q)=\left(\cos \sqrt{\lambda^{2}} \pi\right)^{2} \operatorname{det} A+ & \frac{\lambda}{\sqrt{\lambda^{2}}}\left(\cos \sqrt{\lambda^{2}} \pi \sin \sqrt{\lambda^{2}} \pi\right) \operatorname{trace} A \\
& \quad+\sin ^{2} \sqrt{\lambda^{2}} \pi+O\left(\frac{e^{2 \mid \operatorname{Im} \sqrt{\lambda^{2}}} \mid \pi}{|\lambda|}\right),|\lambda| \rightarrow \infty .
\end{aligned}
$$

The geometric multiplicity and the algebraic multiplicity of the eigenvalues are important for a vectorial Sturm-Liouville equation. For the two-dimensional SturmLiouville equation, Shen proved the following results in $\underline{\mathrm{Sh}}$.

Consider the eigenvalue problem

$$
\left\{\begin{array}{l}
\vec{y}^{\prime \prime}(x)+\left[\lambda I_{2}-Q(x)\right] \vec{y}(x)=\overrightarrow{0}, \\
\vec{y}(0)=\overrightarrow{0}, \vec{y}(\pi)=\overrightarrow{0},
\end{array}\right.
$$


where $Q(x)$ is a two-by-two real symmetric matrix-valued smooth function defined in $[0, \pi], \vec{y}(x)$ is an $\mathbb{R}^{2}$-function defined in the interval $[0, \pi]$, and $\overrightarrow{0}$ is the zero vector in $\mathbb{R}^{2}$. Denote $D_{Q}(\lambda)=\operatorname{det} Y(\pi ; \lambda ; Q)$.

Theorem 2.2. $\lambda_{*}$ is a simple eigenvalue of equation (2.9) if and only if $\lambda_{*}$ is a simple zero of $D_{Q}(\lambda)$.

Theorem 2.3. $\lambda_{*}$ is an eigenvalue of geometric multiplicity two of equation (2.9) if and only if $\lambda_{*}$ is a double zero of $D_{Q}(\lambda)$.

Let $\lambda_{n}$ be an eigenvalue of equation (2.9) and $m_{n}$ be the geometric multiplicity of $\lambda_{n}, n \in \mathbb{N}$. We call the set $\left\{\left(\lambda_{n}, m_{n}\right) \mid n \in \mathbb{N}\right\}$ the spectral set of equation (2.9), and denote it by $\sigma_{D}(Q)$. Denote $K_{Q}(\lambda)=\widehat{Y}(\pi ; \lambda ; Q) Z(\pi ; \lambda ; Q)$, where $\widehat{Y}(\pi ; \lambda ; Q)$ is the adjoint matrix of $Y(\pi ; \lambda ; Q)$. Then Shen proved that $K_{Q}(\lambda)$ is real symmetric for $\lambda \in \mathbb{R}$ and

Theorem 2.4. Suppose $\sigma_{D}\left(Q_{1}\right)=\sigma_{D}\left(Q_{2}\right)$ and $K_{Q_{1}}(\lambda)=K_{Q_{2}}(\lambda)$. Then $Q_{1}(x) \equiv$ $Q_{2}(x)$ in $[0, \pi]$.

\section{The Vectorial Hochstadt PRoblem}

The vectorial Hochstadt problem (2.1) is different from its scalar case studied by Hochstadt in $[\mathrm{H}$. One of the major differences is that if we do not require some extra assumption on the potential function $Q(x)$, then the equation (2.1) may have nonreal eigenvalues (see the example in the Introduction). To avoid the appearance of nonreal eigenvalues, we study the eigenvalue problem (2.1) under the assumption that $Q(x)$ is nonnegative definite for all $x \in[0, \pi]$ in this paper. Let $\lambda_{*}$ be an eigenvalue of equation (2.1), and let $\vec{y}_{*}(x)$ be a corresponding eigenfunction. Then $\vec{y}_{*}(x)=Y\left(x ; \lambda_{*}^{2} ; Q\right){\overrightarrow{y_{*}}}^{\prime}(0)$, and

$$
\left[A Y^{\prime}\left(\pi ; \lambda_{*}^{2} ; Q\right)+\lambda_{*} Y\left(\pi ; \lambda_{*}^{2} ; Q\right)\right] \overrightarrow{y_{*}^{\prime}}(0)=\overrightarrow{0}
$$

Thus $\overrightarrow{y_{*}^{\prime}}(0)$ belongs to the null space of $A Y^{\prime}\left(\pi ; \lambda_{*}^{2} ; Q\right)+\lambda_{*} Y\left(\pi ; \lambda_{*}^{2} ; Q\right)$. In other words, the number of linearly independent eigenfunctions corresponding to $\lambda_{*}$, i.e., the geometric multiplicity of $\lambda_{*}$, is determined by the nullity (i.e., the dimension of the null space) of $A Y^{\prime}\left(\pi ; \lambda_{*}^{2} ; Q\right)+\lambda_{*} Y\left(\pi ; \lambda_{*}^{2} ; Q\right)$. Thus we find that the geometric multiplicity of $\lambda_{*}$ is less than or equal to $n$.

Theorem 3.1. All eigenvalues of equation (2.1) are real.

Proof. Suppose $\lambda$ is an eigenvalue of equation (2.1) and $\vec{y}(x)$ is a corresponding eigenfunction. Then

$$
\left\langle\vec{y}^{\prime \prime}(x), \vec{y}(x)\right\rangle+\left\langle\left[\lambda^{2}-Q(x)\right] \vec{y}(x), \vec{y}(x)\right\rangle=0 .
$$

Integrating both sides of (3.1) from 0 to $\pi$ and using integration by parts, we obtain

$$
\begin{aligned}
& {\left[\left\langle\vec{y}^{\prime}(\pi), \vec{y}(\pi)\right\rangle-\left\langle\vec{y}^{\prime}(0), \vec{y}(0)\right\rangle\right]} \\
& \quad-\int_{0}^{\pi}\left\langle\vec{y}^{\prime}(x), \vec{y}^{\prime}(x)\right\rangle d x+\lambda^{2} \int_{0}^{\pi}\langle\vec{y}(x), \vec{y}(x)\rangle d x=\int_{0}^{\pi}\langle Q(x) \vec{y}(x), \vec{y}(x)\rangle d x .
\end{aligned}
$$

Since

$$
\vec{y}(0)=\overrightarrow{0}, A \vec{y}^{\prime}(\pi)+\lambda \vec{y}(\pi)=\overrightarrow{0}
$$


and $A$ is nonsingular, we have

$$
\begin{aligned}
-\lambda\left\langle A^{-1} \vec{y}(\pi), \vec{y}(\pi)\right\rangle+\lambda^{2} & \int_{0}^{\pi}\langle\vec{y}(x), \vec{y}(x)\rangle d x \\
& =\int_{0}^{\pi}\left\langle\vec{y}^{\prime}(x), \vec{y}^{\prime}(x)\right\rangle d x+\int_{0}^{\pi}\langle Q(x) \vec{y}(x), \vec{y}(x)\rangle d x .
\end{aligned}
$$

Since $Q(x)$ is nonnegative definite in $[0, \pi]$,

$$
\int_{0}^{\pi}\left\langle\vec{y}^{\prime}(x), \vec{y}^{\prime}(x)\right\rangle d x+\int_{0}^{\pi}\langle Q(x) \vec{y}(x), \vec{y}(x)\rangle d x \geq 0 .
$$

Note that (3.2) is a quardratic equation of $\lambda$ with discriminant

$$
\begin{aligned}
& \left(\left\langle A^{-1} \vec{y}(\pi), \vec{y}(\pi)\right\rangle\right)^{2} \\
& \quad+4\left(\int_{0}^{\pi}\langle\vec{y}(x), \vec{y}(x)\rangle d x\right)\left(\int_{0}^{\pi}\left\langle\vec{y}^{\prime}(x), \vec{y}^{\prime}(x)\right\rangle d x+\int_{0}^{\pi}\langle Q(x) \vec{y}(x), \vec{y}(x)\rangle d x\right) \geq 0 .
\end{aligned}
$$

Thus we find that $\lambda$ is real.

Lemma 3.2. 0 is not an eigenvalue of equation (2.1).

Proof. Suppose 0 is an eigenvalue of equation (2.1) and $\vec{y}(x)$ is a corresponding eigenfunction. Then

$$
\left\{\begin{array}{l}
\vec{y}^{\prime \prime}(x)-Q(x) \vec{y}(x)=\overrightarrow{0} \\
\vec{y}(0)=\overrightarrow{0}, \vec{y}^{\prime}(\pi)=\overrightarrow{0}
\end{array}\right.
$$

Dotting equation (3.3) with $\vec{y}(x)$ and integrating it from 0 to $\pi$, we have

$$
\int_{0}^{\pi}\left\langle\vec{y}^{\prime \prime}(x), \vec{y}(x)\right\rangle d x=\int_{0}^{\pi}\langle Q(x) \vec{y}(x), \vec{y}(x)\rangle d x .
$$

Using integration by parts, $\vec{y}(0)=\overrightarrow{0}$, and $\vec{y}^{\prime}(\pi)=\overrightarrow{0},(3.4)$ implies that

$$
\int_{0}^{\pi}\left\langle\vec{y}^{\prime}(x), \vec{y}^{\prime}(x)\right\rangle d x+\int_{0}^{\pi}\langle Q(x) \vec{y}(x), \vec{y}(x)\rangle d x=0 .
$$

Since $Q(x)$ is nonnegative definite for all $x$ in $[0, \pi],(3.5)$ implies that $\vec{y}(x)=\overrightarrow{0}$ for all $x \in[0, \pi]$, which is absurd.

\section{Two-dimensional Vectorial Hochstadt Problem}

In this section, we study the relation between the geometric multiplicity and the algebraic multiplicity of an eigenvalue of equation (2.1) in the two-dimensional case. From now on, we assume that $Q(x)$ is a two-by-two nonnegative definite matrix-valued smooth function defined in $[0, \pi]$. In section 2 , we have seen that the characteristic function $\omega_{A}(\lambda ; Q)$ of equation $(2.1)$ is an entire function of order one with respect to $\lambda$ and is of the form $\operatorname{det}\left(A Y^{\prime}\left(\pi ; \lambda^{2} ; Q\right)+\lambda Y\left(\pi ; \lambda^{2} ; Q\right)\right)$. For a two-by-two matrix $M$, we denote its adjoint matrix by $\widehat{M}$, which has the property that

$$
M \widehat{M}=\widehat{M} M=(\operatorname{det} M) I_{2},
$$

and we denote the conjugate transpose of $M$ by $M^{*}$.

Lemma 4.1. Suppose $\lambda_{*}$ is an eigenvalue of equation (2.1) of geometric multiplicity two. Then $\lambda_{*}$ is a double zero of $\omega_{A}(\lambda ; Q)$. 
Proof. Denote

$$
C_{Q}(\lambda)=A Y^{\prime}\left(\pi ; \lambda^{2} ; Q\right)+\lambda Y\left(\pi ; \lambda^{2} ; Q\right) .
$$

Then (4.1) implies that

$$
\omega_{A}(\lambda ; Q)=\operatorname{det} C_{Q}(\lambda) .
$$

From (4.2), we find

$$
\omega_{A}^{\prime}(\lambda ; Q) I_{2}=C_{Q}^{\prime}(\lambda) \widehat{C}_{Q}(\lambda)+C_{Q}(\lambda) \widehat{C}_{Q}^{\prime}(\lambda) .
$$

Now assume that $\lambda_{*}$ is an eigenvalue of equation (2.1) of geometric multiplicity two. Then

$$
C_{Q}\left(\lambda_{*}\right)=\widehat{C}_{Q}\left(\lambda_{*}\right)=0 .
$$

By (4.3) and (4.4), we find that

$$
\omega_{A}^{\prime}\left(\lambda_{*} ; Q\right)=0 .
$$

In order to show $\lambda_{*}$ is a double zero of $\omega_{A}(\lambda ; Q)$, we need to show $\omega_{A}^{\prime \prime}\left(\lambda_{*} ; Q\right) \neq 0$. Differentiating (4.3) with respect to $\lambda$, we obtain

$$
\omega_{A}^{\prime \prime}(\lambda ; Q) I_{2}=C_{Q}^{\prime \prime}(\lambda) \widehat{C}_{Q}(\lambda)+2 C_{Q}^{\prime}(\lambda) \widehat{C}_{Q}^{\prime}(\lambda)+C_{Q}(\lambda) \widehat{C}_{Q}^{\prime \prime}(\lambda) .
$$

Taking $\lambda=\lambda_{*}$ in (4.5), we find

$$
\omega_{A}^{\prime \prime}\left(\lambda_{*} ; Q\right) I_{2}=2 C_{Q}^{\prime}\left(\lambda_{*}\right) \widehat{C}_{Q}^{\prime}\left(\lambda_{*}\right)=2 \operatorname{det}\left(C_{Q}^{\prime}\left(\lambda_{*}\right)\right) I_{2} .
$$

Thus if $\lambda_{*}$ is an eigenvalue of equation (2.1) of geometric multiplicity two, then

$$
\omega_{A}^{\prime \prime}\left(\lambda_{*} ; Q\right)=2 \operatorname{det}\left(C_{Q}^{\prime}\left(\lambda_{*}\right)\right) .
$$

If we can show $\operatorname{det}\left(C_{Q}^{\prime}\left(\lambda_{*}\right)\right) \neq 0$, then $\omega_{A}^{\prime \prime}\left(\lambda_{*} ; Q\right) \neq 0$. Note that

$$
C_{Q}^{\prime}\left(\lambda_{*}\right)=2 \lambda_{*} A Y_{\nu}^{\prime}\left(\pi ; \lambda_{*}^{2} ; Q\right)+2 \lambda_{*}^{2} Y_{\nu}\left(\pi ; \lambda_{*}^{2} ; Q\right)+Y\left(\pi ; \lambda_{*}^{2} ; Q\right) .
$$

Since

$$
\left\{\begin{array}{l}
Y^{\prime \prime}\left(x ; \lambda^{2} ; Q\right)+\left[\lambda^{2} I_{2}-Q(x)\right] Y\left(x ; \lambda^{2} ; Q\right)=0, \\
Y\left(0 ; \lambda^{2} ; Q\right)=0, Y^{\prime}\left(0 ; \lambda^{2} ; Q\right)=I_{2},
\end{array}\right.
$$

differentiating (4.8) with respect to $\lambda$, we obtain

$$
\left\{\begin{array}{l}
2 \lambda Y_{\nu}^{\prime \prime}\left(x ; \lambda^{2} ; Q\right)+2 \lambda\left[\lambda^{2} I_{2}-Q(x)\right] Y_{\nu}\left(x ; \lambda^{2} ; Q\right)+2 \lambda Y\left(x ; \lambda^{2} ; Q\right)=0, \\
2 \lambda Y_{\nu}\left(0 ; \lambda^{2} ; Q\right)=0,2 \lambda Y_{\nu}^{\prime}\left(0 ; \lambda^{2} ; Q\right)=0 .
\end{array}\right.
$$

As Lemma 3.2 implies $\lambda_{*} \neq 0,(4.9)$ can be expressed as

$$
\left\{\begin{array}{l}
Y_{\nu}^{\prime \prime}\left(x ; \lambda_{*}^{2} ; Q\right)+\left[\lambda_{*}^{2} I_{2}-Q(x)\right] Y_{\nu}\left(x ; \lambda_{*}^{2} ; Q\right)=-Y\left(x ; \lambda_{*}^{2} ; Q\right), \\
Y_{\nu}\left(0 ; \lambda_{*}^{2} ; Q\right)=0, Y_{\nu}^{\prime}\left(0 ; \lambda_{*}^{2} ; Q\right)=0 .
\end{array}\right.
$$

By (4.8), (4.10), and $\lambda_{*} \in \mathbb{R}$, we find

$$
\begin{aligned}
Y^{* \prime \prime}\left(x ; \lambda_{*}^{2} ; Q\right) Y_{\nu}\left(x ; \lambda_{*}^{2} ; Q\right)-Y^{*}\left(x ; \lambda_{*}^{2} ; Q\right) Y_{\nu}^{\prime \prime}( & \left.x ; \lambda_{*}^{2} ; Q\right) \\
& =Y^{*}\left(x ; \lambda_{*}^{2} ; Q\right) Y\left(x ; \lambda_{*}^{2} ; Q\right) .
\end{aligned}
$$


Integrating (4.11) from 0 to $\pi$ with respect to $x$ and using the initial conditions of (4.8) and (4.10), we have

$$
\begin{aligned}
Y^{* \prime}\left(\pi ; \lambda_{*}^{2} ; Q\right) Y_{\nu}\left(\pi ; \lambda_{*}^{2} ; Q\right)-Y^{*}\left(\pi ; \lambda_{*}^{2} ; Q\right) & Y_{\nu}^{\prime}\left(\pi ; \lambda_{*}^{2} ; Q\right) \\
& =\int_{0}^{\pi} Y^{*}\left(x ; \lambda_{*}^{2} ; Q\right) Y\left(x ; \lambda_{*}^{2} ; Q\right) d x .
\end{aligned}
$$

Because

$$
C_{Q}^{*}\left(\lambda_{*}\right)=Y^{* \prime}\left(\pi ; \lambda_{*}^{2} ; Q\right) A+\lambda_{*} Y^{*}\left(\pi ; \lambda_{*}^{2} ; Q\right)=0
$$

we have

$$
Y^{* \prime}\left(\pi ; \lambda_{*}^{2} ; Q\right)=-\lambda_{*} Y^{*}\left(\pi ; \lambda_{*}^{2} ; Q\right) A^{-1} .
$$

Using (4.13), we rewrite (4.12) as

$$
\begin{aligned}
-Y^{*}\left(\pi ; \lambda_{*}^{2} ; Q\right) A^{-1}\left[\lambda_{*} Y_{\nu}\left(\pi ; \lambda_{*}^{2} ; Q\right)+\right. & \left.A Y_{\nu}^{\prime}\left(\pi ; \lambda_{*}^{2} ; Q\right)\right] \\
& =\int_{0}^{\pi} Y^{*}\left(x ; \lambda_{*}^{2} ; Q\right) Y\left(x ; \lambda_{*}^{2} ; Q\right) d x .
\end{aligned}
$$

Claim that $Y\left(\pi ; \lambda_{*}^{2} ; Q\right)$ is nonsingular. Otherwise there is $\vec{v} \in \mathbb{R}^{2}, \vec{v} \neq \overrightarrow{0}$, such that

$$
Y\left(\pi ; \lambda_{*}^{2} ; Q\right) \vec{v}=\overrightarrow{0} .
$$

Then by (4.4), we also have

$$
Y^{\prime}\left(\pi ; \lambda_{*}^{2} ; Q\right) \vec{v}=\overrightarrow{0}
$$

But condition $Y\left(\pi ; \lambda_{*}^{2} ; Q\right) \vec{v}=Y^{\prime}\left(\pi ; \lambda_{*}^{2} ; Q\right) \vec{v}=\overrightarrow{0}$ implies that

$$
Y\left(x ; \lambda_{*}^{2} ; Q\right) \vec{v}=\overrightarrow{0}
$$

for all $x \in[0, \pi]$, which is absurd. Thus $Y\left(\pi ; \lambda_{*}^{2} ; Q\right)$ is nonsingular. Then (4.7) and (4.14) imply

$$
\begin{gathered}
C_{Q}^{\prime}\left(\lambda_{*}\right)=-2 \lambda_{*} A Y^{*-1}\left(\pi ; \lambda_{*}^{2} ; Q\right) \int_{0}^{\pi} Y^{*}\left(x ; \lambda_{*}^{2} ; Q\right) Y\left(x ; \lambda_{*}^{2} ; Q\right) d x+Y\left(\pi ; \lambda_{*}^{2} ; Q\right) \\
=A Y^{*-1}\left(\pi ; \lambda_{*}^{2} ; Q\right)\left[Y^{*}\left(\pi ; \lambda_{*}^{2} ; Q\right) A^{-1} Y\left(\pi ; \lambda_{*}^{2} ; Q\right)\right. \\
\left.-2 \lambda_{*} \int_{0}^{\pi} Y^{*}\left(x ; \lambda_{*}^{2} ; Q\right) Y\left(x ; \lambda_{*}^{2} ; Q\right) d x\right] .
\end{gathered}
$$

By (4.8),

$$
\begin{aligned}
Y^{*}\left(x ; \lambda_{*}^{2} ; Q\right) Y^{\prime \prime}\left(x ; \lambda_{*}^{2} ; Q\right)+\lambda_{*}^{2} Y^{*}\left(x ; \lambda_{*}^{2} ; Q\right) Y\left(x ; \lambda_{*}^{2} ; Q\right) \\
-Y^{*}\left(x ; \lambda_{*}^{2} ; Q\right) Q(x) Y\left(x ; \lambda_{*}^{2} ; Q\right)=0 .
\end{aligned}
$$

Integrating the above equation from 0 to $\pi$, and using integration by parts, we obtain

$$
\begin{aligned}
& \left.Y^{*}\left(x ; \lambda_{*}^{2} ; Q\right) Y^{\prime}\left(x ; \lambda_{*}^{2} ; Q\right)\right|_{0} ^{\pi}-\int_{0}^{\pi} Y^{*^{\prime}}\left(x ; \lambda_{*}^{2} ; Q\right) Y^{\prime}\left(x ; \lambda_{*}^{2} ; Q\right) d x \\
& +\lambda_{*}^{2} \int_{0}^{\pi} Y^{*}\left(x ; \lambda_{*}^{2} ; Q\right) Y\left(x ; \lambda_{*}^{2} ; Q\right) d x-\int_{0}^{\pi} Y^{*}\left(x ; \lambda_{*}^{2} ; Q\right) Q(x) Y\left(x ; \lambda_{*}^{2} ; Q\right) d x=0 .
\end{aligned}
$$


This equation implies

$$
\begin{aligned}
& Y^{*}\left(\pi ; \lambda_{*}^{2} ; Q\right) Y^{\prime}\left(\pi ; \lambda_{*}^{2} ; Q\right)+\lambda_{*}^{2} \int_{0}^{\pi} Y^{*}\left(x ; \lambda_{*}^{2} ; Q\right) Y\left(x ; \lambda_{*}^{2} ; Q\right) d x \\
& =\int_{0}^{\pi} Y^{*^{\prime}}\left(x ; \lambda_{*}^{2} ; Q\right) Y^{\prime}\left(x ; \lambda_{*}^{2} ; Q\right) d x+\int_{0}^{\pi} Y^{*}\left(x ; \lambda_{*}^{2} ; Q\right) Q(x) Y\left(x ; \lambda_{*}^{2} ; Q\right) d x .
\end{aligned}
$$

Since $C_{Q}\left(\lambda_{*}\right)=0$,

$$
Y^{\prime}\left(\pi ; \lambda_{*}^{2} ; Q\right)=-\lambda_{*} A^{-1} Y\left(\pi ; \lambda_{*}^{2} ; Q\right) .
$$

Equation (4.16) can be rewritten as

$$
\begin{aligned}
& Y^{*}\left(\pi ; \lambda_{*}^{2} ; Q\right) A^{-1} Y\left(\pi ; \lambda_{*}^{2} ; Q\right)-\lambda_{*} \int_{0}^{\pi} Y^{*}\left(x ; \lambda_{*}^{2} ; Q\right) Y\left(x ; \lambda_{*}^{2} ; Q\right) d x \\
& =-\frac{1}{\lambda_{*}}\left[\int_{0}^{\pi} Y^{*^{\prime}}\left(x ; \lambda_{*}^{2} ; Q\right) Y^{\prime}\left(x ; \lambda_{*}^{2} ; Q\right) d x+\int_{0}^{\pi} Y^{*}\left(x ; \lambda_{*}^{2} ; Q\right) Q(x) Y\left(x ; \lambda_{*}^{2} ; Q\right) d x\right] .
\end{aligned}
$$

By (4.17), we find

$$
\begin{aligned}
Y^{*}\left(\pi ; \lambda_{*}^{2} ; Q\right) A^{-1} Y\left(\pi ; \lambda_{*}^{2} ; Q\right)-2 \lambda_{*} \int_{0}^{\pi} Y^{*}\left(x ; \lambda_{*}^{2} ; Q\right) Y\left(x ; \lambda_{*}^{2} ; Q\right) d x \\
=-\frac{1}{\lambda_{*}}\left[\int_{0}^{\pi} Y^{* \prime}\left(x ; \lambda_{*}^{2} ; Q\right) Y^{\prime}\left(x ; \lambda_{*}^{2} ; Q\right) d x+\int_{0}^{\pi} Y^{*}\left(x ; \lambda_{*}^{2} ; Q\right) Q(x) Y\left(x ; \lambda_{*}^{2} ; Q\right) d x\right. \\
\left.+\lambda_{*}^{2} \int_{0}^{\pi} Y^{*}\left(x ; \lambda_{*}^{2} ; Q\right) Y\left(x ; \lambda_{*}^{2} ; Q\right) d x\right] .
\end{aligned}
$$

Note that

$$
\int_{0}^{\pi} Y^{* \prime}\left(x ; \lambda_{*}^{2} ; Q\right) Y^{\prime}\left(x ; \lambda_{*}^{2} ; Q\right) d x+\int_{0}^{\pi} Y^{*}\left(x ; \lambda_{*}^{2} ; Q\right) Q(x) Y\left(x ; \lambda_{*}^{2} ; Q\right) d x
$$

is positive definite. Hence

$$
\operatorname{det}\left[Y^{*}\left(\pi ; \lambda_{*}^{2} ; Q\right) A^{-1} Y\left(\pi ; \lambda_{*}^{2} ; Q\right)-2 \lambda_{*} \int_{0}^{\pi} Y^{*}\left(x ; \lambda_{*}^{2} ; Q\right) Y\left(x ; \lambda_{*}^{2} ; Q\right) d x\right] \neq 0 .
$$

By (4.15) and (4.19), we find that $\operatorname{det}\left(C_{Q}^{\prime}\left(\lambda_{*}\right)\right) \neq 0$ since $A$ and $Y^{*}\left(\pi ; \lambda_{*}^{2} ; Q\right)$ are nonsingular. Then (4.6) implies that $\omega_{A}^{\prime \prime}\left(\lambda_{*} ; Q\right) \neq 0$. Hence $\lambda_{*}$ is a zero of $\omega_{A}(\lambda ; Q)$ of multiplicity two if it is an eigenvalue of equation (2.1) of geometric multiplicity two.

The following result is an immediate consequence of Lemma 4.1.

Lemma 4.2. If $\lambda_{*}$ is a simple zero of $\omega_{A}(\lambda ; Q)$, then $\lambda_{*}$ is a simple eigenvalue of equation (2.1).

Proof. Suppose $\lambda_{*}$ is of geometric multiplicity two. Then by Lemma 4.1, $\lambda_{*}$ is a double zero of $\omega_{A}(\lambda ; Q)$. This contradicts the assumption on $\lambda_{*}$. Thus $\lambda_{*}$ is a simple eigenvalue of equation (2.1).

Now we want to consider the converse of Lemma 4.1.

Lemma 4.3. If $\lambda_{*}$ is a simple eigenvalue of equation (2.1), then $\lambda_{*}$ is a simple zero of $\omega_{A}(\lambda ; Q)$. 
Proof. Using the same notation as in Lemma 4.1, we denote

$$
C_{Q}(\lambda)=A Y^{\prime}\left(\pi ; \lambda^{2} ; Q\right)+\lambda Y\left(\pi ; \lambda^{2} ; Q\right) .
$$

Let $R(x ; \lambda)=Y\left(x ; \lambda^{2} ; Q\right) \widehat{C}_{Q}(\lambda)$. Then $R\left(x ; \lambda_{*}\right)$ satisfies the equation

$$
\left\{\begin{array}{l}
R^{\prime \prime}\left(x ; \lambda_{*}\right)+\left[\lambda_{*}^{2} I_{2}-Q(x)\right] R\left(x ; \lambda_{*}\right)=0, \\
R\left(0 ; \lambda_{*}\right)=0, R^{\prime}\left(0 ; \lambda_{*}\right)=\widehat{C}_{Q}\left(\lambda_{*}\right), \\
A R^{\prime}\left(\pi ; \lambda_{*}\right)+\lambda_{*} R\left(\pi ; \lambda_{*}\right)=C_{Q}\left(\lambda_{*}\right) \widehat{C}_{Q}\left(\lambda_{*}\right)=0 .
\end{array}\right.
$$

We also have

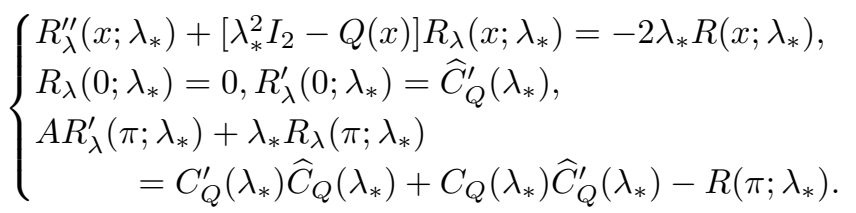

Since Theorem 3.1 implies that $\lambda_{*} \in \mathbb{R}$, we can transform (4.21) into

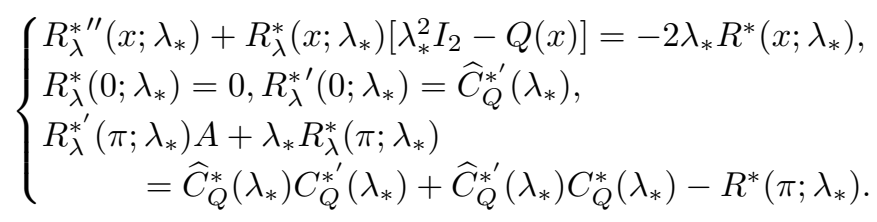

Then by (4.20) and (4.22), we obtain

$$
R_{\lambda}^{*}\left(x ; \lambda_{*}\right) R^{\prime \prime}\left(x ; \lambda_{*}\right)-R_{\lambda}^{* \prime \prime}\left(x ; \lambda_{*}\right) R\left(x ; \lambda_{*}\right)=2 \lambda_{*} R^{*}\left(x ; \lambda_{*}\right) R\left(x ; \lambda_{*}\right) .
$$

Integrating both sides of (4.23) from 0 to $\pi$, and using $R\left(0 ; \lambda_{*}\right)=R_{\lambda}^{*}\left(0 ; \lambda_{*}\right)=0$, we find

$$
R_{\lambda}^{*}\left(\pi ; \lambda_{*}\right) R^{\prime}\left(\pi ; \lambda_{*}\right)-R_{\lambda}^{* \prime}\left(\pi ; \lambda_{*}\right) R\left(\pi ; \lambda_{*}\right)=2 \lambda_{*} \int_{0}^{\pi} R^{*}\left(x ; \lambda_{*}\right) R\left(x ; \lambda_{*}\right) d x .
$$

Applying $A R^{\prime}\left(\pi ; \lambda_{*}\right)+\lambda_{*} R\left(\pi ; \lambda_{*}\right)=0$ in (4.20), (4.24) can be rewritten as

$$
-\left[\lambda_{*} R_{\lambda}^{*}\left(\pi ; \lambda_{*}\right) A^{-1}+R_{\lambda}^{* \prime}\left(\pi ; \lambda_{*}\right)\right] R\left(\pi ; \lambda_{*}\right)=2 \lambda_{*} \int_{0}^{\pi} R^{*}\left(x ; \lambda_{*}\right) R\left(x ; \lambda_{*}\right) d x .
$$

Suppose $\lambda_{*}$ is a zero of $\omega_{A}(\lambda ; Q)$ of multiplicity $\geq 2$. Then

$$
\left.\frac{d\left(\operatorname{det}\left(C_{Q}(\lambda)\right)\right)}{d \lambda}\right|_{\lambda=\lambda_{*}}=0 .
$$

This implies

$$
C_{Q}^{\prime}\left(\lambda_{*}\right) \widehat{C}_{Q}\left(\lambda_{*}\right)+C_{Q}\left(\lambda_{*}\right) \widehat{C}_{Q}^{\prime}\left(\lambda_{*}\right)=0 .
$$

Then by (4.22), we find that

$$
R_{\lambda}^{*^{\prime}}\left(\pi ; \lambda_{*}\right) A+\lambda_{*} R_{\lambda}^{*}\left(\pi ; \lambda_{*}\right)=-R^{*}\left(\pi ; \lambda_{*}\right) .
$$

By (4.25) and (4.26), we find

$$
R^{*}\left(\pi ; \lambda_{*}\right) A^{-1} R\left(\pi ; \lambda_{*}\right)=2 \lambda_{*} \int_{0}^{\pi} R^{*}\left(x ; \lambda_{*}\right) R\left(x ; \lambda_{*}\right) d x .
$$

On the other hand, (4.20) implies

$$
R^{*}\left(x ; \lambda_{*}\right) R^{\prime \prime}\left(x ; \lambda_{*}\right)+R^{*}\left(x ; \lambda_{*}\right)\left[\lambda_{*}^{2} I_{2}-Q(x)\right] R\left(x ; \lambda_{*}\right)=0 .
$$


Integrating the above equation from 0 to $\pi$, and using the boundary conditions of $R\left(x ; \lambda_{*}\right)$, we find

$$
\begin{aligned}
-\lambda_{*} R^{*}\left(\pi ; \lambda_{*}\right) A^{-1} R\left(\pi ; \lambda_{*}\right)+\lambda_{*}^{2} \int_{0}^{\pi} R^{*}\left(x ; \lambda_{*}\right) R\left(x ; \lambda_{*}\right) d x \\
\quad=\int_{0}^{\pi} R^{\prime *}\left(x ; \lambda_{*}\right) R^{\prime}\left(x ; \lambda_{*}\right) d x+\int_{0}^{\pi} R^{*}\left(x ; \lambda_{*}\right) Q(x) R\left(x ; \lambda_{*}\right) d x .
\end{aligned}
$$

Since $\lambda_{*} \neq 0,(4.28)$ implies that

$$
\begin{aligned}
& R^{*}\left(\pi ; \lambda_{*}\right) A^{-1} R\left(\pi ; \lambda_{*}\right)=\lambda_{*} \int_{0}^{\pi} R^{*}\left(x ; \lambda_{*}\right) R\left(x ; \lambda_{*}\right) d x \\
& \quad-\frac{1}{\lambda_{*}}\left[\int_{0}^{\pi} R^{\prime *}\left(x ; \lambda_{*}\right) R^{\prime}\left(x ; \lambda_{*}\right) d x+\int_{0}^{\pi} R^{*}\left(x ; \lambda_{*}\right) Q(x) R\left(x ; \lambda_{*}\right) d x\right] .
\end{aligned}
$$

Comparing (4.29) with (4.27), we obtain

$$
\begin{aligned}
\lambda_{*}^{2} \int_{0}^{\pi} R^{*} & \left(x ; \lambda_{*}\right) R\left(x ; \lambda_{*}\right) d x \\
& =-\left[\int_{0}^{\pi} R^{\prime *}\left(x ; \lambda_{*}\right) R^{\prime}\left(x ; \lambda_{*}\right) d x+\int_{0}^{\pi} R^{*}\left(x ; \lambda_{*}\right) Q(x) R\left(x ; \lambda_{*}\right) d x\right] .
\end{aligned}
$$

Since $\int_{0}^{\pi} R^{*}\left(x ; \lambda_{*}\right) R\left(x ; \lambda_{*}\right) d x, \int_{0}^{\pi} R^{*}\left(x ; \lambda_{*}\right) Q(x) R\left(x ; \lambda_{*}\right) d x, \int_{0}^{\pi} R^{\prime *}\left(x ; \lambda_{*}\right) R^{\prime}\left(x ; \lambda_{*}\right) d x$ are nonnegative definite, the right-hand side of (4.30) is nonpositive definite, while the left-hand side of (4.30) is nonnegative definite, which implies $R\left(x ; \lambda_{*}\right)$ is the zero matrix for all $x$ in $[0, \pi]$. Since $R^{\prime}\left(0 ; \lambda_{*}\right)=\widehat{C}_{Q}\left(\lambda_{*}\right)$, we find $\widehat{C}_{Q}\left(\lambda_{*}\right)$ is the zero matrix, which is absurd. Thus $\lambda_{*}$ is a simple zero of $\omega_{A}(\lambda ; Q)$.

Applying Lemma 4.3, we have the following result.

Lemma 4.4. Suppose $\lambda_{*}$ is a double zero of $\omega_{A}(\lambda ; Q)$. Then $\lambda_{*}$ is also an eigenvalue of equation (2.1) of geometric multiplicity two.

Proof. Suppose $\lambda_{*}$ is a simple eigenvalue of equation (2.1). Then by Lemma 4.3, $\lambda_{*}$ is a simple zero of $\omega_{A}(\lambda: Q)$, which is absurd. Thus $\lambda_{*}$ is of geometric multiplicity two.

Combining Lemmas 4.1-4.4, we obtain the following conclusion.

Theorem 4.5. Let $\lambda_{*}$ be an eigenvalue of equation (2.1). Then the algebraic multiplicity of $\lambda_{*}$ is equal to the geometric multiplicity of $\lambda_{*}$.

We know that $\omega_{A}(\lambda ; Q)$ is an entire function of order one with respect to $\lambda$. Following the theory of Hadamard's factorization, $\omega_{A}(\lambda ; Q)$ can be expressed as an infinite product as

$$
\omega_{A}(\lambda ; Q)=c e^{a \lambda} \prod_{n=-\infty}^{n=\infty}\left(1-\frac{\lambda}{\lambda_{n}}\right)^{m_{n}} e^{m_{n}\left[\frac{\lambda}{\lambda_{n}}+\frac{1}{2}\left(\frac{\lambda}{\lambda_{n}}\right)^{2}+\cdots+\frac{1}{p}\left(\frac{\lambda}{\lambda_{n}}\right)^{p}\right]},
$$

where $\lambda_{n}$ is the eigenvalue of equation (2.1) with respect to $A$ and $Q(x), m_{n}$ is the geometric multiplicity of $\lambda_{n}, n \in \mathbb{N}, p$ is the genus of $\omega_{A}(\lambda ; Q), c$ and $a$ are constants. Note that the genus $p$ is the smallest integer such that $\sum_{n=0}^{\infty}\left(\frac{1}{\lambda_{n}}\right)^{p+1}<\infty$ (see [A]). Since for the order $\rho$ of $\omega_{A}(\lambda ; Q), p \leq \rho \leq p+1$ (see [A]), and $\omega_{A}(\lambda ; Q)$ is 
an entire function of order one, we find that the genus of $\omega_{A}(\lambda ; Q)$ is 0 or 1 . Thus $\omega_{A}(\lambda ; Q)$ can be rewritten by

$$
\omega_{A}(\lambda ; Q)=c e^{a \lambda} \prod_{n=-\infty}^{n=\infty}\left(1-\frac{\lambda}{\lambda_{n}}\right)^{m_{n}} e^{p\left(\frac{m_{n} \lambda}{\lambda_{n}}\right)} .
$$

As stated in section 2 , we call the set $\left\{\left(\lambda_{n}, m_{n}\right) \mid n \in \mathbb{N}\right\}$ the spectral set of equation (2.1), and we denote it by $\sigma_{A}(Q)$.

Theorem 4.6. The characteristic function $\omega_{A}(\lambda ; Q)$ of equation (2.1) is uniquely determined by the spectral set of the equation.

Proof. Suppose there are two functions $Q_{1}(x)$ and $Q_{2}(x)$ with $\sigma_{A}\left(Q_{1}\right)=\sigma_{A}\left(Q_{2}\right)$. Then we have

$$
\begin{aligned}
& \omega_{A}\left(\lambda ; Q_{1}\right)=c_{1} e^{a_{1} \lambda} \prod_{n=-\infty}^{n=\infty}\left(1-\frac{\lambda}{\lambda_{n}}\right)^{m_{n}} e^{p_{1}\left(\frac{m_{n} \lambda}{\lambda_{n}}\right)}, \\
& \omega_{A}\left(\lambda ; Q_{2}\right)=c_{2} e^{a_{2} \lambda} \prod_{n=-\infty}^{n=\infty}\left(1-\frac{\lambda}{\lambda_{n}}\right)^{m_{n}} e^{p_{2}\left(\frac{m_{n} \lambda}{\lambda_{n}}\right)} .
\end{aligned}
$$

Since $\sigma_{A}\left(Q_{1}\right)=\sigma_{A}\left(Q_{2}\right)$, by the definition of genus, we find $p_{1}=p_{2}$. Thus (4.31) and (4.32) imply that for all $\lambda \in \mathbb{C}$,

$$
\frac{\omega_{A}\left(\lambda ; Q_{1}\right)}{\omega_{A}\left(\lambda ; Q_{2}\right)}=\frac{c_{1}}{c_{2}} e^{\left(a_{1}-a_{2}\right) \lambda} .
$$

By the asymptotic formula (2.8), as $|\lambda| \rightarrow \infty$,

$$
\begin{aligned}
& \omega_{A}\left(\lambda ; Q_{1}\right)=\left(\cos \sqrt{\lambda^{2}} \pi\right)^{2} \operatorname{det} A+\frac{\lambda}{\sqrt{\lambda^{2}}}\left(\cos \sqrt{\lambda^{2}} \pi \sin \sqrt{\lambda^{2}} \pi\right) \operatorname{trace} A \\
& +\sin ^{2} \sqrt{\lambda^{2}} \pi+O\left(\frac{e^{2\left|\operatorname{Im} \sqrt{\lambda^{2}}\right| \pi}}{|\lambda|}\right), \\
& \omega_{A}\left(\lambda ; Q_{2}\right)=\left(\cos \sqrt{\lambda^{2}} \pi\right)^{2} \operatorname{det} A+\frac{\lambda}{\sqrt{\lambda^{2}}}\left(\cos \sqrt{\lambda^{2}} \pi \sin \sqrt{\lambda^{2}} \pi\right) \operatorname{trace} A \\
& +\sin ^{2} \sqrt{\lambda^{2}} \pi+O\left(\frac{e^{2\left|\operatorname{Im} \sqrt{\lambda^{2}}\right| \pi}}{|\lambda|}\right) .
\end{aligned}
$$

This implies, for $n \in \mathbb{N}$,

$$
\lim _{n \rightarrow \infty} \frac{\omega_{A}\left(n ; Q_{1}\right)}{\omega_{A}\left(n ; Q_{2}\right)}=1 .
$$

Hence $c_{1}=c_{2}$ and $a_{1}=a_{2}$, i.e., $\omega_{A}\left(\lambda ; Q_{1}\right)=\omega_{A}\left(\lambda ; Q_{2}\right)$.

Let $y_{*}(x)$ be an eigenfunction of equation (2.1) corresponding to the eigenvalue $\lambda_{*}$. Denote

$$
\widetilde{y_{*}}(x)=y_{*}(\pi-x), \widetilde{Q}(x)=Q(\pi-x) .
$$


Then we have

$$
\left\{\begin{array}{l}
\widetilde{y_{*}}{ }^{\prime \prime}(x)+\left[\lambda_{*}^{2} I_{2}-\widetilde{Q}(x)\right] \widetilde{y_{*}}(x)=0, \\
A{\widetilde{y_{*}}}^{\prime}(0)-\lambda_{*} \widetilde{y_{*}}(0)=0, \widetilde{y_{*}}(\pi)=0 .
\end{array}\right.
$$

On the other hand, consider the eigenvalue problem

$$
\left\{\begin{array}{l}
\vec{u}^{\prime \prime}(x)+\left[\lambda^{2} I_{2}-\widetilde{Q}(x)\right] \vec{u}(x)=0, \\
A \vec{u}^{\prime}(0)-\lambda \vec{u}(0)=0, \vec{u}(\pi)=0 .
\end{array}\right.
$$

Let $\vec{u}_{0}\left(x ; \lambda^{2}\right)$ be a solution of the equation

$$
\left\{\begin{array}{l}
\vec{w}^{\prime \prime}(x)+\left[\lambda^{2} I_{2}-\widetilde{Q}(x)\right] \vec{w}(x)=0, \\
A \vec{w}^{\prime}(0)-\lambda \vec{w}(0)=0 .
\end{array}\right.
$$

Then

$$
\vec{u}_{0}\left(x ; \lambda^{2}\right)=Y\left(x ; \lambda^{2} ; \widetilde{Q}\right) \vec{v}_{1}+Z\left(x ; \lambda^{2} ; \widetilde{Q}\right) \vec{v}_{2} .
$$

Following the boundary condition of $u_{0}\left(x ; \lambda^{2}\right)$ at $x=0$ in (4.37), we have

$$
A \vec{v}_{1}-\lambda \vec{v}_{2}=0 .
$$

Equation (4.38) implies that

$$
\vec{u}_{0}\left(x ; \lambda^{2}\right)=\left[\lambda Y\left(x ; \lambda^{2} ; \widetilde{Q}\right) A^{-1}+Z\left(x ; \lambda^{2} ; \widetilde{Q}\right)\right] \vec{v}_{2} .
$$

Thus the eigenvalues and their multiplicity of equation (4.36) are determined by the zeros of the following entire function:

$$
\widetilde{\omega}_{A}(\lambda ; \widetilde{Q})=\operatorname{det}\left[\lambda Y\left(\pi ; \lambda^{2} ; \widetilde{Q}\right) A^{-1}+Z\left(\pi ; \lambda^{2} ; \widetilde{Q}\right)\right] .
$$

Since $A$ is nonsingular, denoting

$$
\widehat{\omega}_{A}(\lambda ; \widetilde{Q})=\operatorname{det}\left[\lambda Y\left(\pi ; \lambda^{2} ; \widetilde{Q}\right)+Z\left(\pi ; \lambda^{2} ; \widetilde{Q}\right) A\right],
$$

we shall call $\widehat{\omega}_{A}(\lambda ; \widetilde{Q})$ the characteristic function of equation (4.36). Denote the spectral set of equation (4.36) by $\widehat{\sigma}_{A}(\widetilde{Q})$, then by the previous observation, we find

$$
\sigma_{A}(Q)=\widehat{\sigma}_{A}(\widetilde{Q}) \text {. }
$$

For the relation between $\omega_{A}(\lambda ; Q)$ and $\widehat{\omega}_{A}(\lambda ; \widetilde{Q})$, we have the following lemma.

Lemma 4.7. $\omega_{A}(\lambda ; Q)=\widehat{\omega}_{A}(\lambda ; \widetilde{Q})$.

Proof. Since $\omega_{A}(\lambda ; Q)$ and $\widehat{\omega}_{A}(\lambda ; \widetilde{Q})$ are entire functions of order one with respect to $\lambda$, and $\sigma_{A}(Q)=\widehat{\sigma}_{A}(\widetilde{Q})$, Hadamard's factorization formulae for $\omega_{A}(\lambda ; Q)$ and $\widehat{\omega}_{A}(\lambda ; \widetilde{Q})$ imply that for all $\lambda \in \mathbb{C}$,

$$
\frac{\omega_{A}(\lambda ; Q)}{\widehat{\omega}_{A}(\lambda ; \widetilde{Q})}=\frac{c e^{a \lambda}}{\widehat{c} e^{\widehat{a} \lambda}}
$$

where $c, \widehat{c}, a, \widehat{a}$ are constants. On the other hand, by (2.4) and (2.6),

$$
\begin{aligned}
\widehat{\omega}_{A}(\lambda ; \widetilde{Q})=\left(\cos \sqrt{\lambda^{2}} \pi\right)^{2} \operatorname{det} A+ & \frac{\lambda}{\sqrt{\lambda^{2}}}\left(\cos \sqrt{\lambda^{2}} \pi \sin \sqrt{\lambda^{2}} \pi\right) \operatorname{trace} A \\
& +\sin ^{2} \sqrt{\lambda^{2}} \pi+O\left(\frac{e^{2 \mid \operatorname{Im} \sqrt{\lambda^{2}}} \pi}{|\lambda|}\right),|\lambda| \rightarrow \infty .
\end{aligned}
$$


Using (4.40) and (2.8), for $n \in \mathbb{N}$, we find that

$$
\lim _{n \rightarrow \infty} \frac{\omega_{A}(n ; Q)}{\widehat{\omega}_{A}(n ; \widetilde{Q})}=\lim _{n \rightarrow \infty} \frac{c e^{a n}}{\widehat{c} e^{\widehat{a} n}}=1 .
$$

This leads to $c=\widehat{c}$ and $a=\widehat{a}$. Hence

$$
\omega_{A}(\lambda ; Q)=\widehat{\omega}_{A}(\lambda ; \widetilde{Q}) .
$$

Next we study the problem about how many spectra of equation (2.1) can determine $Q(x)$ uniquely. Let $A_{1}, A_{2}$, and $A_{3}$ be three two-by-two nonsingular real symmetric matrices, writing

$$
A_{j}=\left(\begin{array}{cc}
\alpha_{j} & \beta_{j} \\
\beta_{j} & \gamma_{j}
\end{array}\right), j=1,2,3
$$

We say that $A_{1}, A_{2}$, and $A_{3}$ are linearly independent if the column vectors $\left(\alpha_{j}, \beta_{j}, \gamma_{j}\right), j=1,2,3$, are linearly independent over $\mathbb{R}$. Recall that in [Sh], Shen introduced a function $K_{Q}(\lambda)=\widehat{Y}(\pi ; \lambda ; Q) Z(\pi ; \lambda ; Q)$ and showed that $K_{Q}(\lambda)$ and $\sigma_{D}(Q)$ determine $Q(x)$ uniquely. To obtain our goal, we only need find how many boundary conditions can determine $K_{Q}(\lambda)$ and $\sigma_{D}(Q)$ uniquely.

Consider the following example: Let $Q_{0}(x)$ be a two-by-two nonnegative definite matrix-valued function defined in $[0, \pi]$ and

$$
A_{1}=\left(\begin{array}{cc}
a & 0 \\
0 & a
\end{array}\right), A_{2}=\left(\begin{array}{ll}
0 & b \\
b & 0
\end{array}\right)
$$

where $a, b \neq 0$. Consider the eigenvalue problem

$$
\left\{\begin{array}{l}
\vec{y}^{\prime \prime}(x)+\left[\lambda^{2} I_{2}-Q_{0}(x)\right] \vec{y}(x)=\overrightarrow{0}, \\
\vec{y}(0)=\overrightarrow{0}, A_{j} \vec{y}^{\prime}(\pi)+\lambda \vec{y}(\pi)=\overrightarrow{0}, j=1,2,
\end{array}\right.
$$

where

$$
Q_{0}(x)=\left(\begin{array}{ll}
p(x) & r(x) \\
r(x) & q(x)
\end{array}\right)
$$

Denote

$$
\check{Q}_{0}(x)=\left(\begin{array}{ll}
q(x) & r(x) \\
r(x) & p(x)
\end{array}\right) .
$$

Suppose $\vec{y}_{*}(x)$ is an eigenfunction of (4.41) corresponding to $\lambda_{*}$. Denoting

$$
\check{\vec{y}}_{*}(x)=\left(\begin{array}{ll}
0 & 1 \\
1 & 0
\end{array}\right) \vec{y}_{*}(x)
$$

we find

$$
\left\{\begin{array}{l}
\check{\vec{y}}_{*}^{\prime \prime}(x)+\left[\lambda_{*}^{2} I_{2}-\check{Q}_{0}(x)\right] \check{\vec{y}}_{*}(x)=\overrightarrow{0}, \\
\check{\vec{y}}_{*}(0)=\overrightarrow{0}, A_{j} \overrightarrow{\breve{y}}_{*}^{\prime}(\pi)+\lambda \overrightarrow{\vec{y}}_{*}(\pi)=\overrightarrow{0}, j=1,2 .
\end{array}\right.
$$

From (4.41) and (4.42), we obtain that $\sigma_{A_{1}}\left(Q_{0}\right)=\sigma_{A_{1}}\left(\check{Q}_{0}\right)$ and $\sigma_{A_{2}}\left(Q_{0}\right)=\sigma_{A_{2}}\left(\check{Q}_{0}\right)$. Thus two spectra of equation (2.1) corresponding to two linearly independent nonsingular real symmetric matrices $A_{1}$ and $A_{2}$ cannot determine the potential function $Q(x)$ uniquely. In order to determine the potential function $Q(x)$ uniquely, we need more than two spectra. 
Theorem 4.8. Let $Q_{1}(x)$ and $Q_{2}(x)$ be two two-by-two nonnegative definite matrixvalued smooth functions defined in $[0, \pi]$, and let $A_{1}, A_{2}$, and $A_{3}$ be two-by-two nonsingular real symmetric matrices. Suppose $A_{1}, A_{2}$, and $A_{3}$ are linearly independent, and

$$
\widehat{\omega}_{A_{j}}\left(\lambda ; Q_{1}\right)=\widehat{\omega}_{A_{j}}\left(\lambda ; Q_{2}\right), j=1,2,3 .
$$

Then $\operatorname{det}\left(Y\left(\pi ; \lambda^{2} ; Q_{1}\right)\right)=\operatorname{det}\left(Y\left(\pi ; \lambda^{2} ; Q_{2}\right)\right), K_{Q_{1}}\left(\lambda^{2}\right)=K_{Q_{2}}\left(\lambda^{2}\right)$, and $Q_{1}(x)=$ $Q_{2}(x)$.

Proof. Denote

$$
\begin{aligned}
& D_{1}\left(\lambda^{2}\right)=\operatorname{det}\left(Y\left(\pi ; \lambda^{2} ; Q_{1}\right)\right)=\operatorname{det}\left(\widehat{Y}\left(\pi ; \lambda^{2} ; Q_{1}\right)\right), \\
& D_{2}\left(\lambda^{2}\right)=\operatorname{det}\left(Y\left(\pi ; \lambda^{2} ; Q_{2}\right)\right)=\operatorname{det}\left(\widehat{Y}\left(\pi ; \lambda^{2} ; Q_{2}\right)\right) .
\end{aligned}
$$

By the assumptions, we find

$$
D_{1}\left(\lambda^{2}\right) D_{2}\left(\lambda^{2}\right) \widehat{\omega}_{A_{j}}\left(\lambda ; Q_{1}\right)=D_{1}\left(\lambda^{2}\right) D_{2}\left(\lambda^{2}\right) \widehat{\omega}_{A_{j}}\left(\lambda ; Q_{2}\right), j=1,2,3 .
$$

Since for $k=1,2$,

$$
\begin{aligned}
D_{k}\left(\lambda^{2}\right) \widehat{\omega}_{A_{j}}\left(\lambda ; Q_{k}\right) & =\operatorname{det}\left(\widehat{Y}\left(\pi ; \lambda^{2} ; Q_{k}\right)\right) \widehat{\omega}_{A_{j}}\left(\lambda ; Q_{k}\right) \\
& =\operatorname{det}\left[\lambda D_{k}\left(\lambda^{2}\right) I_{2}+K_{Q_{k}}\left(\lambda^{2}\right) A_{j}\right],
\end{aligned}
$$

from (4.43), we find for $j=1,2,3$,

$$
D_{2}\left(\lambda^{2}\right) \operatorname{det}\left[\lambda D_{1}\left(\lambda^{2}\right) I_{2}+K_{Q_{1}}\left(\lambda^{2}\right) A_{j}\right]=D_{1}\left(\lambda^{2}\right) \operatorname{det}\left[\lambda D_{2}\left(\lambda^{2}\right) I_{2}+K_{Q_{2}}\left(\lambda^{2}\right) A_{j}\right] .
$$

Calculating the determinants, we obtain

$$
\begin{aligned}
& D_{2}\left(\lambda^{2}\right)\left[\lambda^{2} D_{1}^{2}\left(\lambda^{2}\right)+\operatorname{det}\left(K_{Q_{1}}\left(\lambda^{2}\right) A_{j}\right)+\lambda D_{1}\left(\lambda^{2}\right) \operatorname{trace}\left(K_{Q_{1}}\left(\lambda^{2}\right) A_{j}\right)\right] \\
& \quad=D_{1}\left(\lambda^{2}\right)\left[\lambda^{2} D_{2}^{2}\left(\lambda^{2}\right)+\operatorname{det}\left(K_{Q_{2}}\left(\lambda^{2}\right) A_{j}\right)+\lambda D_{2}\left(\lambda^{2}\right) \operatorname{trace}\left(K_{Q_{2}}\left(\lambda^{2}\right) A_{j}\right)\right],
\end{aligned}
$$

where $j=1,2,3$. Comparing the odd part (resp., the even part) of both sides of equation (4.44) with respect to $\lambda$, we find

$$
\begin{aligned}
\lambda^{2} D_{1}^{2}\left(\lambda^{2}\right) D_{2}\left(\lambda^{2}\right)+D_{2}\left(\lambda^{2}\right) & \operatorname{det}\left(K_{Q_{1}}\left(\lambda^{2}\right) A_{j}\right) \\
= & \lambda^{2} D_{1}\left(\lambda^{2}\right) D_{2}^{2}\left(\lambda^{2}\right)+D_{1}\left(\lambda^{2}\right) \operatorname{det}\left(K_{Q_{2}}\left(\lambda^{2}\right) A_{j}\right),
\end{aligned}
$$

and

$$
\lambda D_{1}\left(\lambda^{2}\right) D_{2}\left(\lambda^{2}\right) \operatorname{trace}\left(K_{Q_{1}}\left(\lambda^{2}\right) A_{j}\right)=\lambda D_{1}\left(\lambda^{2}\right) D_{2}\left(\lambda^{2}\right) \operatorname{trace}\left(K_{Q_{2}}\left(\lambda^{2}\right) A_{j}\right) .
$$

Denote

$$
A_{j}=\left(\begin{array}{cc}
\alpha_{j} & \beta_{j} \\
\beta_{j} & \gamma_{j}
\end{array}\right), j=1,2,3
$$

Noting that for $\lambda \in \mathbb{R}, K_{Q_{1}}\left(\lambda^{2}\right)$ and $K_{Q_{2}}\left(\lambda^{2}\right)$ are real symmetric (see [Sh]), we write

$$
K_{Q_{1}}\left(\lambda^{2}\right)=\left(\begin{array}{ll}
k_{11}^{(1)}\left(\lambda^{2}\right) & k_{12}^{(1)}\left(\lambda^{2}\right) \\
k_{12}^{(1)}\left(\lambda^{2}\right) & k_{22}^{(1)}\left(\lambda^{2}\right)
\end{array}\right), K_{Q_{2}}\left(\lambda^{2}\right)=\left(\begin{array}{ll}
k_{11}^{(2)}\left(\lambda^{2}\right) & k_{12}^{(2)}\left(\lambda^{2}\right) \\
k_{12}^{(2)}\left(\lambda^{2}\right) & k_{22}^{(2)}\left(\lambda^{2}\right)
\end{array}\right) .
$$

Since (4.46) implies that

$$
\operatorname{trace}\left(K_{Q_{1}}\left(\lambda^{2}\right) A_{j}\right)=\operatorname{trace}\left(K_{Q_{2}}\left(\lambda^{2}\right) A_{j}\right),
$$

from (4.47), we find for $\lambda \in \mathbb{R}$,

(4.48) $k_{11}^{(1)}\left(\lambda^{2}\right) \alpha_{j}+2 k_{12}^{(1)}\left(\lambda^{2}\right) \beta_{j}+k_{22}^{(1)}\left(\lambda^{2}\right) \gamma_{j}=k_{11}^{(2)}\left(\lambda^{2}\right) \alpha_{j}+2 k_{12}^{(2)}\left(\lambda^{2}\right) \beta_{j}+k_{22}^{(2)}\left(\lambda^{2}\right) \gamma_{j}$. 
Since $A_{1}, A_{2}$, and $A_{3}$ are linearly independent, the matrix

$$
\left(\begin{array}{lll}
\alpha_{1} & \beta_{1} & \gamma_{1} \\
\alpha_{2} & \beta_{2} & \gamma_{2} \\
\alpha_{3} & \beta_{3} & \gamma_{3}
\end{array}\right)
$$

is invertible. Then by (4.48), we find for all $\lambda \in \mathbb{R}$,

$$
k_{11}^{(1)}\left(\lambda^{2}\right)=k_{11}^{(2)}\left(\lambda^{2}\right), k_{12}^{(1)}\left(\lambda^{2}\right)=k_{12}^{(2)}\left(\lambda^{2}\right), k_{22}^{(1)}\left(\lambda^{2}\right)=k_{22}^{(2)}\left(\lambda^{2}\right) .
$$

Hence for all $\lambda \in \mathbb{R}$,

$$
K_{Q_{1}}\left(\lambda^{2}\right)=K_{Q_{2}}\left(\lambda^{2}\right) .
$$

As $k_{11}^{(l)}, k_{12}^{(l)}, k_{22}^{(l)}$ are entire functions in $\lambda^{2}, l=1,2$, we conclude that $K_{Q_{1}}\left(\lambda^{2}\right)=$ $K_{Q_{2}}\left(\lambda^{2}\right)$ for all $\lambda \in \mathbb{C}$.

Now denote

$$
k\left(\lambda^{2}\right)=\operatorname{det}\left(K_{Q_{1}}\left(\lambda^{2}\right)\right)=\operatorname{det}\left(K_{Q_{2}}\left(\lambda^{2}\right)\right), a_{j}=\operatorname{det} A_{j} .
$$

Then (4.45) can be expressed as

(4.50) $\lambda^{2} D_{1}^{2}\left(\lambda^{2}\right) D_{2}\left(\lambda^{2}\right)+D_{2}\left(\lambda^{2}\right) \cdot a_{j} \cdot k\left(\lambda^{2}\right)=\lambda^{2} D_{1}\left(\lambda^{2}\right) D_{2}^{2}\left(\lambda^{2}\right)+D_{1}\left(\lambda^{2}\right) \cdot a_{j} \cdot k\left(\lambda^{2}\right)$.

From (4.50), we find that

$$
\left[\lambda^{2} D_{1}\left(\lambda^{2}\right) D_{2}\left(\lambda^{2}\right)-a_{j} k\left(\lambda^{2}\right)\right]\left[D_{1}\left(\lambda^{2}\right)-D_{2}\left(\lambda^{2}\right)\right]=0 .
$$

Since $D_{1}\left(\lambda^{2}\right), D_{2}\left(\lambda^{2}\right), k\left(\lambda^{2}\right)$ are entire functions in $\lambda^{2}$, (4.51) tells us either

$$
\lambda^{2} D_{1}\left(\lambda^{2}\right) D_{2}\left(\lambda^{2}\right)-a_{j} k\left(\lambda^{2}\right) \equiv 0
$$

or

$$
D_{1}\left(\lambda^{2}\right)-D_{2}\left(\lambda^{2}\right) \equiv 0
$$

Suppose

$$
\lambda^{2} D_{1}\left(\lambda^{2}\right) D_{2}\left(\lambda^{2}\right)-a_{j} k\left(\lambda^{2}\right) \equiv 0 .
$$

Then as $k\left(\lambda^{2}\right)=D_{1}\left(\lambda^{2}\right) \operatorname{det}\left(Z\left(\pi ; \lambda^{2} ; Q_{1}\right)\right)$, (4.52) implies that

$$
\lambda^{2} D_{2}\left(\lambda^{2}\right)-a_{j} \operatorname{det}\left(Z\left(\pi ; \lambda^{2} ; Q_{1}\right)\right) \equiv 0 .
$$

As (2.4) and (2.6) imply that

$$
\lambda^{2} D_{2}\left(\lambda^{2}\right)=\sin ^{2} \sqrt{\lambda^{2}} \pi+O\left(\frac{e^{2\left|\operatorname{Im} \sqrt{\lambda^{2}}\right| \pi}}{|\lambda|}\right)
$$

and

$$
\operatorname{det}\left(Z\left(\pi ; \lambda^{2} ; Q_{1}\right)\right)=\cos ^{2} \sqrt{\lambda^{2}} \pi+O\left(\frac{e^{2\left|\operatorname{Im} \sqrt{\lambda^{2}}\right| \pi}}{|\lambda|}\right),
$$

using (4.54) and (4.55), we find for $j=1,2,3, n \in \mathbb{N}$,

$$
\lim _{n \rightarrow \infty}\left[n^{2} D_{2}\left(n^{2}\right)-a_{j} \operatorname{det}\left(Z\left(\pi ; n^{2} ; Q_{1}\right)\right)\right]=-a_{j} \neq 0,
$$

which contradicts (4.53). Thus we find that $D_{1}\left(\lambda^{2}\right)-D_{2}\left(\lambda^{2}\right) \equiv 0$. Since $D_{1}\left(\lambda^{2}\right)=$ $D_{2}\left(\lambda^{2}\right)$ and $K_{Q_{1}}\left(\lambda^{2}\right)=K_{Q_{2}}\left(\lambda^{2}\right)$, by Theorem 2.5, $Q_{1}(x)=Q_{2}(x)$. 
Applying Theorem 4.8, we obtain the following result.

Theorem 4.9. Let $Q_{1}(x)$ and $Q_{2}(x)$ be two two-by-two nonnegative definite matrixvalued smooth functions defined in $[0, \pi]$, and let $A_{1}, A_{2}$, and $A_{3}$ be two-by-two nonsingular real symmetric matrices. Suppose $A_{1}, A_{2}$, and $A_{3}$ are linearly independent, and $\sigma_{A_{j}}\left(Q_{1}\right)=\sigma_{A_{j}}\left(Q_{2}\right), j=1,2,3$. Then $Q_{1}(x) \equiv Q_{2}(x)$ in $[0, \pi]$.

Proof. Since $\sigma_{A_{j}}\left(Q_{1}\right)=\sigma_{A_{j}}\left(Q_{2}\right), j=1,2,3$, we know

$$
\omega_{A_{j}}\left(\lambda ; Q_{1}\right)=\omega_{A_{j}}\left(\lambda ; Q_{2}\right) .
$$

Then Lemma 4.7 implies that for $j=1,2,3$,

$$
\widehat{\omega}_{A_{j}}\left(\lambda ; \widetilde{Q}_{1}\right)=\widehat{\omega}_{A_{j}}\left(\lambda ; \widetilde{Q}_{2}\right) .
$$

By (4.56), Theorem 4.8 implies that

$$
\begin{aligned}
\operatorname{det}\left(Y\left(\pi ; \lambda^{2} ; \widetilde{Q}_{1}\right)\right) & =\operatorname{det}\left(Y\left(\pi ; \lambda^{2} ; \widetilde{Q}_{2}\right)\right), \\
K_{\widetilde{Q}_{1}}\left(\lambda^{2}\right) & =K_{\widetilde{Q}_{2}}\left(\lambda^{2}\right),
\end{aligned}
$$

and

$$
\widetilde{Q}_{1}(x)=\widetilde{Q}_{2}(x) .
$$

Hence $Q_{1}(x) \equiv Q_{2}(x)$ in $[0, \pi]$.

\section{REFERENCES}

[A] Ahlfors, L. V., 1979, Complex Analysis (New York: McGraw-Hill). MR510197 (80c:30001)

[GS] Gesztesy, F. and Simon, B., 2000, A new approach to the inverse spectral theory, II. General real potentials and the connection to the spectral measure Annals of math 152 593-643. MR.1804532 (2001m:34185b)

[H] Hochstadt, H., 1967, On the inverse problems associated with second-order differential operators, Acta Mathematica 119 173-192. MR0223633 (36:6681)

[PT] Pöschel, J. and Trubowitz, E., 1987, Inverse Spectral Theory, (New York: Academic). MR894477 (89b:34061)

[Sh] Shen, C.-L., 2001, Some inverse spectral problems for vectorial Sturm-Liouville equations, Inverse Problems 17 1253-1294. MR1862190(2002g:34025)

[Si] Simon, B., 1999, A new approach to the inverse spectral theory, I. Fundamental formalism, Annals of Math (2), 150 1029-1057. MR1740987(2001m:34185a)

Department of Mathematics, Tsing Hua University, Hsinchu, Taiwan 30043, Republic OF CHINA

E-mail address: d917201@oz.nthu.edu.tw 